\section{Síndrome de Burnout em graduandos de Odontologia}

\section{Burnout syndrome among dental students}

Juliana Alvares Duarte Bonini Campos'

Paula Cristina Jordani"

Miriane Lucindo Zucoloto"

Fernanda Salloume Sampaio Bonafé"

João Maroco"'I

' Departamento de Odontologia Social da Faculdade de Odontologia de Araraquara da Universidade Estadual Paulista - UNESP.

"Curso de graduação em Odontologia da Faculdade de Odontologia de Araraquara da Universidade Estadual Paulista - UNESP.

II' Instituto Superior de Psicologia Aplicada - ISPA, Lisboa, Portugal.

\section{Resumo}

A Síndrome de Burnout caracteriza-se por esgotamento profissional e tem sido relatada em estudantes universitários. O objetivo desse estudo foi estimar a prevalência da Síndrome de Burnout em estudantes de Odontologia de uma universidade pública e sua relação com características sociodemográficas. Todos os estudantes ( $\mathrm{n}=300$ ) foram convidados a participar. Utilizou-se o Inventário de Burnout de Maslach - versão estudantes (MBI-SS). Realizou-se análise das propriedades psicométricas do MBI-SS. Para comparação entre os escores médios das dimensões do Burnout realizou-se Análise de Variância Multivariada (MANOVA) seguida de Análise de Variância (ANOVA) e testes post-hoc de Tukey. Dos 235 participantes, $72,8 \%$ eram mulheres e a média de idade era de 21,0 \pm 1,8 anos. O MBI-SS mostrou-se confiável e válido. Dos estudantes, 17,0\% apresentaram a Síndrome de Burnout. Verificou-se relação significativa entre a Síndrome de Burnout e o desempenho do estudante no curso ( $\mathrm{F}=$ 4,433, p < 0,001), o consumo de medicação devido aos estudos $(\mathrm{F}=7,721, \mathrm{p}<0,001)$ e o pensamento de desistir do curso $(F=16,168$, $\mathrm{p}<0,001)$. Foram mais acometidos aqueles com desempenho ruim no curso, que consomem medicações devido aos estudos e que já pensaram em desistir do curso. Conclui-se que a prevalência da Síndrome entre os estudantes de Odontologia foi alta, sendo significativa a relação entre a Síndrome e o desempenho do estudante no curso, o consumo de medicação devido aos estudos e o pensamento de desistir do curso.

Palavras-chave: Estudantes. Esgotamento profissional. Odontologia. Saúde Pública. 


\section{Abstract}

The burnout syndrome is characterized by professional exhaustion and has been reported in college students. The aim of this study was to estimate the prevalence of Burnout Syndrome among dentistry students from a public university, and its relationship to socio-demographic characteristics. All students $(n=300)$ were invited to participate. We used the Maslach Burnout Inventory - Student Version (MBI-SS). We carried out an analysis of the MBI-SS' psychometric properties. Multivariate Analysis of Variance (MANOVA) was performed, followed by Analysis of Variance (ANOVA) and Tukey's post-hoc tests to compare the mean scores of burnout dimensions. Of the 235 participants, $72.8 \%$ were women and the mean age was $21.0 \pm 1.8$ years. The MBI-SS was reliable and valid. Of the students, $17.0 \%$ had Burnout Syndrome. There was a significant relation between Burnout Syndrome and a student's performance during the course $(\mathrm{F}=4.433$, $\mathrm{p}<0.001$ ), medication intake because of studies $(\mathrm{F}=7.721, \mathrm{p}<0.001)$, and the thought of dropping the course $(\mathrm{F}=16.168$, $\mathrm{p}<0.001)$. The students most affected were those with poor performance, those who took medication because of studies, and those with thoughts of dropping the course. We concluded that the prevalence of the syndrome among dentistry students was high, with a significant relation between the syndrome and a student's academic performance, use of medication because of studies, and thoughts of dropping the course.

Keywords: Students. Professional fatigue. Odontology. Public Health.

\section{Introdução}

A Síndrome de Burnout é definida por Maslach e Jackson ${ }^{1}$ como uma Síndrome do meio laboral caracterizada por um processo de resposta de cronificação ao estresse ocupacional, quando os métodos de enfrentamento falham ou são insuficientes, trazendo consigo consequências negativas tanto no âmbito individual, como profissional, familiar e social. É referida como uma síndrome multidimensional constituída por exaustão emocional, desumanização e reduzida realização no trabalho.

De acordo com Batista et al. ${ }^{6}$, a síndrome de burnout é uma questão de saúde pública devido às suas implicações para a saúde física, mental e social dos indivíduos. Salanova e Llorens ${ }^{7}$ destacam que, atualmente, o Burnout é um dos agravos ocupacionais de caráter psicossocial mais importantes na sociedade.

Incialmente, essa síndrome foi descrita em profissionais cujo contato emocional com pessoas é intenso ${ }^{1-4}$; entretanto, mais recentemente, o conceito de Burnout foi expandido para todos os grupos ocupacionais, incluindo estudantes ${ }^{5}$. Nestes últimos, pode-se citar os trabalhos de Schaufeli et al. ${ }^{5}$, McManus et al. ${ }^{8}$, Willcock et al. ${ }^{9}$, Carlotto et al. ${ }^{10}$, Ried et al. ${ }^{11}$, Garrosa et al. ${ }^{4}$, Barboza et al. ${ }^{12}$, Maroco et al. ${ }^{13}$, Dyrbye et al. ${ }^{14}$ e Zhang et al. ${ }^{15}$. De modo geral, esses trabalhos afirmam que é de suma importância a investigação e monitoramento desta Síndrome desde o ingresso no ensino superior, pois é nesse momento que podem surgir os primeiros sintomas de Burnout e sua detecção precoce pode ser uma estratégia interessante para planejamento e execução de medidas preventivas e de enfrentamento.

Estudos na área odontológica são escassos, Pohlmann et al. ${ }^{16}$ investigaram a síndrome de Burnout em graduandos do quarto e quinto ano de Odontologia de faculdades da Alemanha e Suíça e observaram que aproximadamente um terço dos estudantes sofria de despersonalização. Humphris et al. ${ }^{17}$ realizaram um estudo com graduandos do primeiro ano de Odontologia em sete 
faculdades da Europa e verificaram alto nível de exaustão emocional. Sofola e Jeboda ${ }^{18}$ investigaram a origem do estresse em graduandos de Odontologia da Universidade de Lagos, Nigéria, e verificaram que o improviso do sistema de educação odontológica foi o maior fator de estresse apontado.

Com relação a estudos brasileiros que investigaram a Síndrome de Burnout em estudantes de Odontologia, encontrou-se apenas um trabalho conduzido por Carlotto et al. ${ }^{10}$. Entretanto, a amostra foi composta por estudantes de oito áreas da saúde e a apresentação dos resultados não foi conduzida separadamente por área do conhecimento, impossibilitando a análise da síndrome separadamente para os estudantes de Odontologia. Assim, sabendo da importância do conhecimento dessa condição nessa população para adequação de ações visando a melhoria de sua qualidade de vida e rendimento escolar, este estudo foi realizado com o objetivo verificar e caracterizar a presença da Síndrome de Burnout em graduandos de um curso de Odontologia.

\section{Métodos}

\section{População de Estudo}

Todos os estudantes de graduação da Faculdade de Odontologia de Araraquara - UNESP ( $n=300)$, matriculados no ano de 2009, foram convidados a participar.

\section{Variáveis de Estudo}

Para caracterização da população, um questionário sociodemográfico foi aplicado abordando aspectos como sexo, idade, ano do curso, necessidade de recorrer a professores particulares, ordem de preferência pelo curso no vestibular, expectativas e desempenho no curso, opinião sobre as condições materiais da faculdade, com quem o estudante mora, consumo de medicação devido aos estudos e pensamento de desistir do curso.

A versão em português* do Inventário de Burnout Maslach - Student Survey (MBI-SS) foi utilizada para a avaliação da Síndrome de Burnout. O MBI-SS é uma escala de autoavaliação tipo Likert de sete pontos cujas categorias de resposta vão de "nunca" a "todos os dias". O instrumento consiste de 15 questões que se subdividem em três dimensões, Exaustão Emocional (EE) composta por 5 itens, Descrença (DE) com 4 itens e Eficácia Profissional (EP) com 6 itens.

\section{Procedimentos}

Os questionários foram aplicados a todos os estudantes em sala de aula em semanas sem avaliações em um momento previamente acordado com o professor, que estava ciente quanto ao fornecimento de 15 minutos de sua aula teórica para aplicação dos mesmos.

\section{Aspectos Éticos}

Participaram do estudo apenas aqueles estudantes que concordaram com os termos do Consentimento Livre e Esclarecido. A realização desse estudo foi aprovada pelo Comitê de Ética em Pesquisa da Faculdade de Odontologia (protocolo n.06/09) e não há conflito de interesses.

\section{Estudo Piloto}

Foi realizado um estudo piloto para calibração intra-examinador. Participaram dessa etapa 100 estudantes voluntários que receberam o MBI-SS para preenchimento em 2 momentos distintos com intervalo de 1 semana entre os mesmos para estudo de sua reprodutibilidade. Para análise dos dados foi utilizado o Coeficiente de Correlação Intraclasse ( $\rho$ ) por ponto e por intervalo de $95 \%$ de confiança $\left(\mathrm{IC}_{95 \%}\right)$. Foi

\footnotetext{
* Nesse estudo, foi utilizada a versão em português do MBI-SS elaborada por Campos et al. ${ }^{19}$, em consonância com o novo acordo ortográfico estabelecido entre os países de língua portuguesa. Cabe esclarecer que, para confecção dessa versão, utilizou-se a versão em português proposta por Maroco et al. ${ }^{13}$ em Portugal e a proposta por Carlotto et al. ${ }^{20}$, no Brasil. Essa nova versão passou pelas etapas de validação facial, de conteúdo e teve sua validade fatorial e confiabilidade aferidas em estudo anterior.
} 
estimada também a consistência interna do inventário utilizando o Coeficiente alpha de Cronbach $(\alpha)$ padronizado para cada dimensão.

\section{Análise das Qualidades Psicométricas}

A análise das qualidades psicométricas do MBI-SS foi realizada seguindo as orientações de Maroco $^{21}$. Assim, a análise da validade de construto e da confiabilidade foi efetuada por intermédio de uma análise fatorial confirmatória. Como medidas de qualidade de ajustamento foram utilizados os índices $\chi^{2} / g l$, CFI, GFI e RMSEA. Considerou-se, de acordo com os critérios de Maroco $^{21}$, que o modelo tem validade fatorial quando o $\chi^{2} / \mathrm{gl}$ encontra-se entre 1 e 2 , CFI e GFI $\geq 0,9$ e RMSEA $<0,08$. A validade convergente dos fatores foi avaliada pela Variância extraída média (VEM) para cada fator. Considerou-se que os fatores apresentam validade convergente quando a VEM é superior a 0,5. Finalmente, a confiabilidade do construto foi avaliada por meio da confiabilidade composta (CC) e da consistência interna $(\alpha)$. Valores de CC e Coeficiente $\alpha$ de Cronbach superiores a 0,7 indicam boa fiabilidade.

\section{Análise Estatística}

Os dados foram organizados e apurados para todas variáveis de estudo com o auxílio do programa STATA 9.0. Como ponto de corte para determinação da Exaustão e Descrença utilizou-se o percentil 66 (P66) e para Eficácia Profissional o percentil 33 (P33) conforme proposta de Maslach e Jackson $^{22}$. Foi caracterizado como portador da Síndrome de Burnout aquele indivíduo que apresentou simultaneamente valores médios acima do P66 para Exaustão e Descrença e abaixo do P33 para Eficácia Profissional.

A prevalência de Burnout foi estimada por ponto e por intervalo de $95 \%$ de confiança. Para estudo de comparação entre os escores médios das dimensões do Burnout realizou-se Análise de Variância
Multivariada (MANOVA) com as 3 dimensões do Burnout como variáveis dependentes e as variáveis sociodemográficas como variáveis independentes. Onde a MANOVA revelou um efeito estatisticamente significativo dos fatores, procedeu-se para cada um deles a Análise de Variância (ANOVA) seguida pelos testes post-hoc de Tukey. Os pressupostos da MANOVA foram avaliados com os testes de Shapiro-Wilk univariados e M de Box, não se observando violações severas destes pressupostos que comprometam a validade das conclusões estatísticas. O nível de significância adotado para tomada de decisão foi de $5 \%$.

\section{Resultados}

Dos 100 participantes do estudo piloto, um foi descartado pelo fato do preenchimento dos questionários não ter sido adequado. A reprodutibilidade das dimensões Exaustão $\left(\rho=0,84, \mathrm{IC}_{95 \%}=0,77-0,89\right)$ e Descrença $\left(\rho=0,71, \mathrm{IC}_{95 \%}=0,59-0,79\right)$ pode ser considerada boa enquanto para a Eficácia Profissional $\left(\rho=0,65, \mathrm{IC}_{95 \%}=0,52\right.$ 0,75 ) a concordância foi moderada. Do mesmo modo, no estudo piloto, a consistência interna das duas primeiras dimensões foi excelente ( $\left.\alpha_{\text {Exaustão }}=0,87 ; \alpha_{\text {Descrença }}=0,81\right)$ enquanto para Eficácia profissional $(\alpha=0,65)$ esta esteve abaixo do valor desejado $(0,70)$.

No estudo definitivo, a taxa de resposta foi de 78,3\%, assim, participaram 235 estudantes sendo que $171(72,0 \%)$ eram mulheres e a média de idade foi de 21,0 anos (DP = 1,8).

A distribuição dos graduandos segundo suas características sócio-demográficas encontra-se na Tabela 1.

Nem todos os estudantes preencheram todas as questões. A maioria dos estudantes escolheu o curso de Odontologia como primeira opção, nunca recorreram a professores particulares, considera o curso melhor em relação as suas expectativas iniciais, afirma ter um bom desempenho no curso, classifica os professores como competentes e mora com amigos. Apesar de a maior parte dos estudantes afirmar nunca ter tomado 
Tabela 1 - Distribuição $(n(\%))$ dos graduandos de Odontologia participantes quanto às características sociodemográficas. Araraquara, 2009.

Table 1 - Distribution (n(\%)) of undergraduate dental students as to socio-demographic characteristics. Araraquara, 2009.

\begin{tabular}{|c|c|}
\hline Característica & $\mathrm{n}(\%)$ \\
\hline \multicolumn{2}{|l|}{ Ano do curso } \\
\hline Primeiro & $43(18,62)$ \\
\hline Segundo & $56(24,25)$ \\
\hline Terceiro & $56(24,24)$ \\
\hline Quarto & $75(32,89)$ \\
\hline \multicolumn{2}{|l|}{ Professor particular } \\
\hline Sim & $36(15,38)$ \\
\hline Não & $198(84,62)$ \\
\hline \multicolumn{2}{|c|}{ Ordem de preferência pelo curso } \\
\hline 1a opção & $168(71,79)$ \\
\hline $2^{\mathrm{a}}$ opção & $57(24,36)$ \\
\hline$\geq 3^{\mathrm{a}}$ opção & $9(3,85)$ \\
\hline \multicolumn{2}{|c|}{ Expectativas iniciais em relação ao curso } \\
\hline Muito pior & $3(1,28)$ \\
\hline Pior & $14(5,96)$ \\
\hline Igual & $50(21,28)$ \\
\hline Melhor & $116(49,36)$ \\
\hline Muito melhor & $52(22,13)$ \\
\hline \multicolumn{2}{|l|}{ Desempenho no curso } \\
\hline Ruim & $4(1,70)$ \\
\hline Regular & $48(20,43)$ \\
\hline Bom & $169(71,91)$ \\
\hline Excelente & $14(5,96)$ \\
\hline \multicolumn{2}{|c|}{ Desempenho dos professores } \\
\hline Muito incompetentes & $3(1,28)$ \\
\hline Incompetentes & $1(0,43)$ \\
\hline Razoáveis & $21(8,97)$ \\
\hline Competentes & $154(65,81)$ \\
\hline Muito competentes & $55(23,50)$ \\
\hline \multicolumn{2}{|c|}{ Condições materiais da faculdade } \\
\hline Muito más & $1(0,43)$ \\
\hline Más & - \\
\hline Razoáveis & $34(14,53)$ \\
\hline Boas & $124(52,99)$ \\
\hline Muito boas & $75(32,05)$ \\
\hline \multicolumn{2}{|l|}{ Com quem você vive } \\
\hline Sozinho & $61(26,29)$ \\
\hline Família & $55(23,71)$ \\
\hline Amigos, colegas & $116(50,00)$ \\
\hline \multicolumn{2}{|c|}{ Quem financia seus estudos } \\
\hline Família & $219(96,48)$ \\
\hline Bolsa & $8(3,52)$ \\
\hline \multicolumn{2}{|c|}{ Já precisou tomar medicação devido aos estudos } \\
\hline Nunca & $142(60,94)$ \\
\hline As vezes & $77(33,05)$ \\
\hline Com frequência & $14(6,01)$ \\
\hline \multicolumn{2}{|c|}{ Já pensou em desistir do curso } \\
\hline Não & $139(59,66)$ \\
\hline As vezes & $80(34,33)$ \\
\hline Com frequência & $14(6,01)$ \\
\hline
\end{tabular}


medicação devido aos estudos e nunca ter pensado em desistir do curso, esses comportamentos podem ser observados em aproximadamente $40,0 \%$ dos participantes.

Na Figura 1 apresenta-se a Análise fatorial confirmatória do MBI-SS para a amostra em estudo.

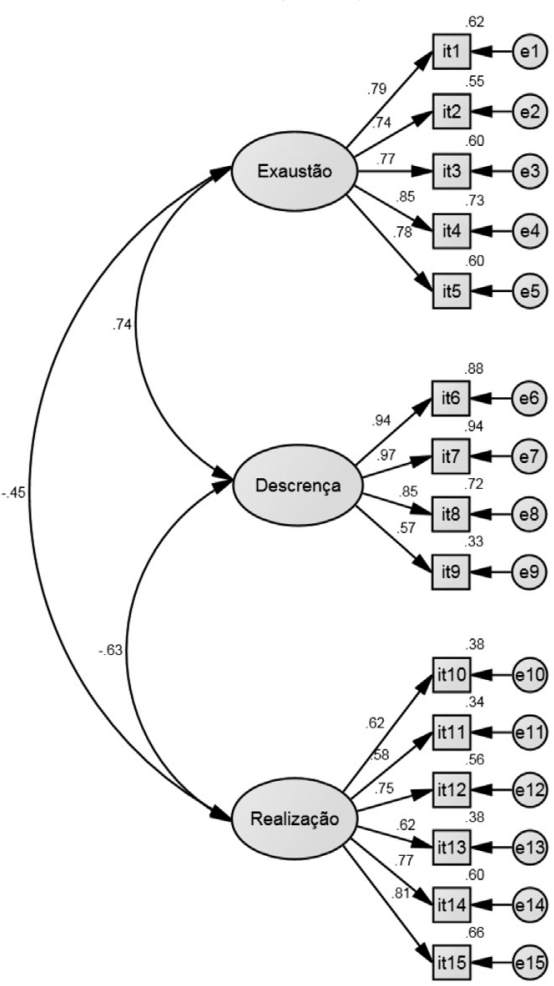

Figura 1 - Análise Fatorial Confirmatória da versão em português do MBI-SS $\left(\chi^{2} / \mathrm{DF}=2,30\right.$; $\mathrm{CFI}=0,95 ; \mathrm{GFI}=0,90 ; \mathrm{RMSEA}=0,07$ ).

Figure 1 - Confirmatory Factor Analysis of the Portuguese version of the MBI-SS (SS $\left(\chi^{2} / d f=\right.$ 2.30; $C F I=0.95 ; G F I=0.90 ; R M S E A=0.07)$.

O ajustamento do modelo trifatorial na presente amostra, a validade convergente $\left(\operatorname{VEM}_{\text {Exaustão }}=0,62 ; \operatorname{VEM}_{\text {Descrençă }}=0,72\right.$; $\mathrm{VEM}_{\text {Efićácia Profissional }}=0,50$ ) e a confiabilidade do MBI-SS (Exaustão: $\mathrm{CC}=0,89 ; \alpha=0,89$; Descrença: CC $=0,91 ; \alpha=0,90$; Eficácia Profissional: $\mathrm{CC}=0,85 ; \alpha=0,85$ ) foram adequados.

A distribuição das respostas dos participantes que responderam o MBI-SS encontra-se na Tabela 2.

Observa-se que $47,2 \%$ dos indivíduos sentem-se emocionalmente esgotados pelos estudos, 43,8\% quando levantam, $57,9 \%$ acreditam que é um grande esforço estudar e frequentar as aulas e $45,5 \%$ sentem-se consumidos pelos estudos. Dos estudantes, $75,3 \%$ afirmaram ter perdido interesse nos estudos e $68,9 \%$ estão descrentes quanto aos seus potenciais e da utilidade dos estudos.

A Síndrome de Burnout foi identificada em $17,0 \%\left(\mathrm{IC}_{95 \%}=13,0-21,0 \%\right)$ dos estudantes.

A comparação entre os escores médios obtidos nas diferentes dimensões do MBI SS segundo as variáveis de interesse estão apresentadas na Tabela 3 .

Nota-se relação significativa entre o acometimento pelo Burnout e o desempenho no curso, o consumo de medicações devido aos estudos e a possibilidade de desistir do curso, sendo mais acometidos aqueles com desempenho ruim no curso, que consomem medicações devido aos estudos e que já pensaram em desistir do curso. Os indivíduos do sexo masculino apresentaram escore médio de Eficácia Profissional significativamente menor que as mulheres. Os estudantes do primeiro e terceiro ano do curso de graduação apresentaram escores de Exaustão significativamente maiores que os demais.

\section{Discussão}

O reconhecimento da síndrome de Burnout como um problema de saúde pública levou à inserção da mesma na lista de Doenças profissionais relacionadas ao trabalho ${ }^{23}$. Esse fato exprime a intensa preocupação com as consequências físicas, emocionais e sociais advindas da instalação do Burnout e alerta para a necessidade de realização de estudos científicos que investiguem a prevalência da síndrome e os fatores a ela associados em diferentes populações.

Os cirurgiões-dentistas, devido às peculiaridades de sua profissão, tais como o contato com o paciente, fatores financeiros, postura específica de trabalho e atuação com espaço restrito à cavidade bucal, fazem 
Tabela 2 - Distribuição das respostas dos participantes (n(\%)) ao MBI-SS. Araraquara, 2009.

Table 2 - Distribution of the participants' answers (\%) on the MBI-SS. Araraquara, 2009.

\begin{tabular}{|c|c|c|c|c|c|c|c|}
\hline \multirow[t]{2}{*}{ Pergunta } & \multicolumn{7}{|c|}{ Resposta } \\
\hline & sempre & $\begin{array}{l}\text { quase } \\
\text { sempre }\end{array}$ & $\begin{array}{l}\text { muitas } \\
\text { vezes }\end{array}$ & regularmente & $\begin{array}{l}\text { algumas } \\
\text { vezes }\end{array}$ & $\begin{array}{l}\text { quase } \\
\text { nunca }\end{array}$ & nunca \\
\hline $\begin{array}{l}\text { 1- Sinto-me emocionalmente } \\
\text { esgotado pelos meus estudos }\end{array}$ & $18(7,7)$ & $35(14,9)$ & $58(24,7)$ & $39(16,6)$ & $34(14,5)$ & $42(17,9)$ & $9(3,8)$ \\
\hline $\begin{array}{l}\text { 2- Sinto-me esgotado no fim de um } \\
\text { dia que tenho aula }\end{array}$ & $4(1,7)$ & $16(6,8)$ & $47(20,0)$ & $37(15,7)$ & $47(20,0)$ & $64(27,2)$ & $20(8,5)$ \\
\hline $\begin{array}{l}\text { 3- Sinto-me cansado quando me } \\
\text { levanto para enfrentar outro dia de } \\
\text { aula }\end{array}$ & $13(5,5)$ & $41(17,5)$ & $49(20,8)$ & $46(19,6)$ & $39(16,6)$ & $36(15,3)$ & $11(4,7)$ \\
\hline $\begin{array}{l}\text { 4- Estudar e frequentar as aulas são, } \\
\text { para mim, um grande esforço }\end{array}$ & $38(16,27)$ & $51(21,7)$ & $47(20,0)$ & $34(14,5)$ & $31(13,2)$ & $24(10,2)$ & $10(4,3)$ \\
\hline $\begin{array}{l}\text { 5- Sinto-me consumido pelos meus } \\
\text { estudos }\end{array}$ & $27(11,5)$ & $34(14,5)$ & $46(19,6)$ & $38(16,2)$ & $34(14,5)$ & $38(16,2)$ & $18(7,7)$ \\
\hline $\begin{array}{l}\text { 6- Tenho me tornado menos } \\
\text { interessado nos meus estudos desde } \\
\text { que entrei nesta universidade }\end{array}$ & $88(37,5)$ & $52(22,1)$ & $35(14,9)$ & $23(9,8)$ & $14(6,0)$ & $15(6,4)$ & $8(3,4)$ \\
\hline $\begin{array}{l}\text { 7- Tenho me tornado menos } \\
\text { interessado nos meus estudos }\end{array}$ & $79(33,6)$ & $60(25,5)$ & $38(16,2)$ & $21(8,9)$ & $17(7,2)$ & $12(5,1)$ & $8(3,4)$ \\
\hline $\begin{array}{l}\text { 8- Tenho estado mais descrente do } \\
\text { meu potencial e da utilidade dos } \\
\text { meus estudos }\end{array}$ & $74(31,5)$ & $53(22,6)$ & $35(14,9)$ & $33(14,0)$ & $14(6,0)$ & $17(7,2)$ & $9(3,8)$ \\
\hline $\begin{array}{l}\text { 9- Eu questiono o sentido e a } \\
\text { importância de meus estudos }\end{array}$ & $69(29,4)$ & $54(23,0)$ & $30(12,8)$ & $32(13,6)$ & $21(8,9)$ & $15(6,4)$ & $14(6,0)$ \\
\hline $\begin{array}{l}\text { 10- Posso resolver os problemas que } \\
\text { surgem nos meus estudos }\end{array}$ & $50(21,3)$ & $86(36,6)$ & $29(12,3)$ & $39(16,6)$ & $20(8,5)$ & $9(3,8)$ & $2(0,8)$ \\
\hline $\begin{array}{l}\text { 11- Acredito que eu seja eficaz na } \\
\text { contribuição das aulas que freqüento }\end{array}$ & $27(11,5)$ & $73(31,2)$ & $34(14,5)$ & $36(15,4)$ & $34(14,5)$ & $24(10,3)$ & $6(2,6)$ \\
\hline 12-Considero-me um bom estudante & $36(15,5)$ & $85(36,5)$ & $39(16,7)$ & $40(17,2)$ & $22(9,4)$ & $8(3,4)$ & $3(1,3)$ \\
\hline $\begin{array}{l}\text { 13- Sinto-me estimulado quando } \\
\text { concluo com êxito a minha meta de } \\
\text { estudos }\end{array}$ & $112(47,9)$ & $59(25,2)$ & $22(9,4)$ & $18(7,7)$ & $10(4,3)$ & $8(3,4)$ & $5(2,4)$ \\
\hline $\begin{array}{l}\text { 14- Tenho aprendido muitas coisas } \\
\text { interessantes no decorrer dos meus } \\
\text { estudos }\end{array}$ & $86(36,6)$ & $67(28,5)$ & $41(17,5)$ & $26(11,1)$ & $11(4,7)$ & $3(1,3)$ & $1(0,4)$ \\
\hline $\begin{array}{l}\text { 15- Durante as aulas, sinto-me } \\
\text { confiante: realizo minhas tarefas de } \\
\text { forma eficaz }\end{array}$ & $28(11,9)$ & $64(27,2)$ & $51(21,7)$ & $49(20,8)$ & $22(9,4)$ & $16(6,8)$ & $5(2,1)$ \\
\hline
\end{tabular}

parte de uma categoria de profissionais da saúde que podem apresentar alto nível de estresse ${ }^{17,18}$. A literatura tem apontado que a cronificação desse estresse, aliado a fatores como falta de energia e entusiasmo, sensação de esgotamento, insatisfação no trabalho e instabilidade emocional podem levar ao desenvolvimento da Síndrome de Burnout.É possivel que o densenvolvimento da síndrome possa ocorrer precocemente, já desde o aprendizado da profissão, e que esse fato possa potencializar o acometimento futuro dos profissionais.

Para o enfrentamento dessa situação, verifica-se a necessidade de identificação precoce de sintomas relacionados ao Burnout, o que deve ocorrer preferencialmente quando os profissionais ainda estão em fase de formação, ou seja, durante sua graduação $0^{5,10,14,15,18}$. Segundo Martinez et 
Tabela 3 - Estudo de comparação entre as dimensões componentes do MBI-SS e as variáveis de interesse. Araraquara, 2009.

Table 3 - Comparison of MBI-SS factors and sociodemographic variables of interest. Araraquara, 2009.

\begin{tabular}{|c|c|c|c|c|c|c|}
\hline \multirow[t]{2}{*}{ Característica } & \multicolumn{6}{|c|}{ Média \pm desvio-padrão } \\
\hline & Exaustão & $\mathrm{p}$ & Descrença & $\mathrm{p}$ & $\begin{array}{c}\text { Eficácia } \\
\text { Profissional }\end{array}$ & $\mathrm{p}$ \\
\hline \multicolumn{7}{|l|}{ Sexo } \\
\hline Masculino & $2,87 \pm 1,33$ & & $1,76 \pm 1,52$ & & $4,14 \pm 1,21$ & \\
\hline Feminino & $2,93 \pm 1,41$ & 0,55 & $1,65 \pm 1,51$ & 0,96 & $4,37 \pm 1,05$ & 0,45 \\
\hline \multicolumn{7}{|l|}{ Ano do curso } \\
\hline Primeiro & $3,39 \pm 1,31$ & & $1,90 \pm 1,32$ & & $4,13 \pm 0,99$ & \\
\hline Segundo & $2,74 \pm 1,33$ & & $1,49 \pm 1,38$ & & $4,32 \pm 1,24$ & \\
\hline Terceiro & $2,86 \pm 1,37$ & & $1,75 \pm 1,54$ & & $4,36 \pm 1,13$ & \\
\hline Quarto & $2,82 \pm 1,44$ & 0,08 & $1,65 \pm 1,69$ & 0,45 & $4,35 \pm 1,05$ & 0,67 \\
\hline \multicolumn{7}{|c|}{ Professores particulares } \\
\hline Sim & $2,98 \pm 1,46$ & & $1,64 \pm 1,53$ & & $4,32 \pm 1,22$ & \\
\hline Não & $2,90 \pm 1,37$ & 0,83 & $1,69 \pm 1,51$ & 0,66 & $4,30 \pm 1,08$ & 0,73 \\
\hline \multicolumn{7}{|c|}{ Ordem de preferência pelo curso } \\
\hline $1^{\text {a }}$ opção & $2,82 \pm 1,32$ & & $1,51 \pm 1,37$ & & $4,46 \pm 1,01$ & \\
\hline Outra & $3,15 \pm 1,51$ & $0,04^{*}$ & $2,13 \pm 1,76$ & $0,01^{*}$ & $3,89 \pm 1,23$ & $0,01^{*}$ \\
\hline \multicolumn{7}{|c|}{ Expectativas iniciais em relação ao curso } \\
\hline Pior & $4,44^{a} \pm 1,19$ & & $3,18^{\mathrm{a}} \pm 1,66$ & & $3,61^{\mathrm{a}} \pm 1,56$ & \\
\hline Igual & $2,94^{b} \pm 1,39$ & & $2,10^{b} \pm 1,77$ & & $4,03^{\mathrm{a}} \pm 1,02$ & \\
\hline Melhor & $2,76^{b} \pm 1,32$ & $0,01^{*}$ & $1,41^{c} \pm 1,30$ & $0,01^{*}$ & $4,45^{b} \pm 1,04$ & $0,01^{*}$ \\
\hline \multicolumn{7}{|c|}{ Desempenho no curso } \\
\hline Ruim & $5,10^{\mathrm{a}} \pm 0,93$ & & $4,81^{a} \pm 1,21$ & & $1,50^{\mathrm{a}} \pm 0,56$ & \\
\hline Regular & $3,40^{b} \pm 1,29$ & & $2,24^{b} \pm 1,61$ & & $3,73^{b} \pm 0,97$ & \\
\hline Bom & $2,77^{\complement} \pm 1,37$ & & $1,52^{\mathrm{c}} \pm 1,35$ & & $4,48^{\mathrm{c}} \pm 0,96$ & \\
\hline Excelente & $2,37^{c} \pm 1,08$ & $0,01^{*}$ & $0,88^{\mathrm{c}} \pm 1,62$ & $0,01^{*}$ & $4,87^{c} \pm 1,35$ & $0,01^{*}$ \\
\hline \multicolumn{7}{|c|}{ Competência dos professores } \\
\hline Incompetentes & $4,13^{a} \pm 1,60$ & & $2,75^{\mathrm{a}} \pm 2,46$ & & $2,83^{\mathrm{a}} \pm 1,43$ & \\
\hline Razoáveis & $3,58^{\mathrm{a}} \pm 1,40$ & & $2,57^{a} \pm 1,44$ & & $3,78^{a} \pm 1,31$ & \\
\hline Competentes & $2,83^{b} \pm 1,36$ & $0,01^{*}$ & $1,58^{\mathrm{b}} \pm 1,48$ & $0,01^{*}$ & $4,38^{b} \pm 1,05$ & $0,01^{*}$ \\
\hline \multicolumn{7}{|c|}{ Como você classificaria as condições materiais de sua escola } \\
\hline Razoáveis & $2,92 \pm 1,51$ & & $2,10 \pm 1,70$ & & $4,16 \pm 1,08$ & \\
\hline Boas & $2,91 \pm 1,37$ & 0,73 & $1,62 \pm 1,47$ & 0,22 & $4,33 \pm 1,11$ & 0,58 \\
\hline \multicolumn{7}{|l|}{ Com quem você vive } \\
\hline Sozinho & $2,91 \pm 1,52$ & & $1,85 \pm 1,73$ & & $4,23 \pm 1,13$ & \\
\hline Família & $2,89 \pm 1,37$ & & $1,69 \pm 1,51$ & & $4,27 \pm 1,14$ & \\
\hline Amigos, colegas & $2,93 \pm 1,33$ & 0,98 & $1,59 \pm 1,39$ & 0,76 & $4,36 \pm 1,07$ & 0,71 \\
\hline \multicolumn{7}{|c|}{ Quem financia seus estudos } \\
\hline Família & $2,89 \pm 1,37$ & & $1,65 \pm 1,46$ & & $4,33 \pm 1,08$ & \\
\hline Bolsa & $3,74 \pm 1,77$ & 0,22 & $2,61 \pm 2,60$ & 0,27 & $3,57 \pm 1,49$ & 0,11 \\
\hline \multicolumn{7}{|c|}{ Você já precisou tomar algum tipo de medicação devido aos estudos } \\
\hline Não & $2,63 \pm 1,31$ & & $1,53 \pm 1,38$ & & $4,41 \pm 1,01$ & \\
\hline Sim & $3,36 \pm 1,38$ & $0,01^{*}$ & $1,93 \pm 1,68$ & $0,02^{*}$ & $4,13 \pm 1,23$ & $0,04^{*}$ \\
\hline \multicolumn{7}{|c|}{ Já pensou em desistir do curso } \\
\hline Não & $2,59 \pm 1,27$ & & $1,09 \pm 1,10$ & & $4,64 \pm 0,95$ & \\
\hline Sim & $3,43 \pm 1,40$ & $0,01^{*}$ & $2,63 \pm 1,60$ & $0,01^{*}$ & $3,77 \pm 1,21$ & $0,01^{*}$ \\
\hline
\end{tabular}

* diferença estatística significativa para $a=0,05 ;$ a,b,c letras iguais indicam similaridade estatística/* Statistically significant difference at $5 \%$; ${ }^{a, b, c}$ equal letters indicate statistical similarity 
al. ${ }^{24}$, a detecção precoce de níveis sintomáticos de Burnout pode ser um bom indicador de possíveis dificuldades, tanto no êxito escolar quanto profissional, possibilitando intervenções preventivas e elaboração de medidas de enfrentamento. Assim, abre-se a necessidade da realização de estudos de rastreamento para identificação da prevalência da Síndrome de Burnout e de seus fatores associados.

Para tanto faz-se necessária a utilização de instrumentos de medida confiáveis e válidos. A considerar que as características psicométricas de um instrumento estão intrinsicamente relacionadas à amostra de estudo ${ }^{19,25,26}$, essa avaliação deveria ser realizada antes da apresentação dos resultados de qualquer estudo epidemiológico que envolva aplicação de escalas, uma vez que essa é a única maneira de se avaliar a qualidade dos dados obtidos. Entretanto, apesar da relevância dessa análise, essa conduta não tem sido recorrente em estudos epidemiológicos. O MBI-SS apresentou excelente validade convergente e consistência interna. A dimensão eficácia profissional apresentou consistência ligeiramente menor o que corrobora com dados da literatura ${ }^{9,11,18}$. O adequado ajuste do modelo tri-fatorial aponta para boa validade fatorial do MBI-SS (Figura 1). Em conjunto com a validade convergente, consistencia interna e validade divergente, esses dados suportam a validade do constructo no MBISS na presente amostra.

A prevalência da Síndrome de Burnout entre os graduandos de Odontologia de Araraquara pode ser considerada alta. Pöhlmann et al. ${ }^{16}$ também encontraram altos escores de Burnout em estudantes de Odontologia e salientam os altos níveis de Descrença encontrados, o que segundo os autores pode ser reflexo da insegurança dos estudantes para lidar com pacientes. Por outro lado, Carlotto et al. ${ }^{10}$ não encontraram a presença da Síndrome nos estudantes, tendo encontrado apenas valores elevados de Exaustão, o que, segundo o modelo processual de Burnout de Maslasch, é o primeiro indicativo de desenvolvimento de Burnout no futuro. Esse fato, segundo Pöhlmann et al. ${ }^{16}$, pode ser justificado pela ansiedade dos estudantes diante das avaliações, do pouco tempo para lazer e diante do estresse sofrido na transição para a fase clínica.

A possibilidade de desistir do curso esteve significativamente associada às dimensões do MBI-SS, o que vai ao encontro dos resultados obtidos por Carlotto et al. ${ }^{10}$. Para explicar esse fato pode-se citar o argumento apresentado por Batista et al. ${ }^{6}$, quando da investigação da síndrome de Burnout em professores, que afirmam que a intenção de abandono da atividade laboral pode ser considerada uma tentativa de lidar com a exaustão emocional decorrente muitas vezes do desequilíbrio entre os investimentos realizados e as recompensas recebidas.

Neste estudo, foram verificadas ainda outras relações significativas entre a ocorrência de Burnout e variáveis sociodemográficas em estudantes que, tanto quanto sabemos, não foram ainda apontadas na literatura. Nestas se incluem o desempenho do estudante no curso, o pensamento de desistir do curso e a ingestão de medicação associada aos estudos. A relação significativa entre o desempenho no curso e as dimensões do MBI-SS aponta para um maior acometimento de Burnout naqueles estudantes cujo desempenho é ruim, o que pode ocorrer pelo fato de as atividades relacionadas ao curso se tornarem uma tarefa mais desgastante para esses alunos e propiciarem uma atitude de ceticismo.

É possivel que a ingestão de medicação relacionada com os estudos possa ser uma consequência do aparecimento da Sindrome de Burnout. Contudo, salienta-se que esta sugestão deve ser interpretada com cautela, uma vez que este estudo é de natureza transversal e correlacional.

Observou-se também relação significativa entre o ano do curso e a dimensão Exaustão do MBI-SS. Martinez et al. ${ }^{24} \mathrm{e}$ Carlotto et al. ${ }^{10}$ também verificaram essa relação, afirmando que quanto mais inicial a fase escolar, maior a exaustão emocional e a descrença com o ensino e menor o sentimento de eficácia profissional, e justificam 
esse fato diante da necessidade de os estudantes recém-saídos do ensino médio necessitarem lidar com uma nova realidade e precisarem adotar uma postura de maior autonomia e responsabilidade. Contudo, na Tabela 3 pode-se observar que os maiores escores foram encontrados tanto para os estudantes do primeiro quanto do terceiro ano e somente para a dimensão Exaustão.

Apesar de a Síndrome de Burnout não se apresentar significativamente relacionada ao sexo, observou-se diferença estatística significativa nos escores de Eficácia profissional, sendo que os homens apresentaram valores mais baixos. Alemany Martinez et al. $^{24}$ encontraram maior prevalência da Síndrome de Burnout e de seus componentes nos indivíduos do sexo masculino. Os autores justificam esse achado diante do fato de as mulheres procurarem ajuda e suporte familiar com maior frequência que os homens.

Os dados apresentados apontam para uma relação entre os componentes da Síndrome de Burnout e as variáveis sociodemográficas, o que sinaliza para a importância de medidas de intervenção junto às variáveis laborais e psicossociais que podem influenciar no adoecimento ocupacional dos estudantes de Odontologia. Entretanto, deve-se destacar a possibilidade de ter ocorrido nesse estudo um viés de seleção, uma vez que, os estudantes participantes foram voluntários e que, portanto, existe a possibilidade de aqueles estudantes mais afetados não terem participado. Assim, os escores médios de exaustão e descrença poderiam ser mais elevados e os de eficácia profissional mais baixos que aqueles apresentados. A par dessa limitação, espera-se que este estudo possa auxiliar a comunidade escolar na identificação da síndrome de Burnout e de seus fatores associados na busca de uma melhor qualidade de vida e aproveitamento escolar.

\section{Conclusão}

A prevalência da Síndrome de Burnout entre os estudantes de Odontologia foi de $17 \%$. Verificou-se relação significativa entre a Síndrome e o desempenho do estudante no curso, o consumo de medicação devido aos estudos e a existência do pensamento de desistir do curso.

\section{Agradecimentos}

À FAPESP pelo financiamento do estudo (processo: 2009/03447-0).

\section{Referências}

1. Maslach C, Jackson SE. The Measurement of Experienced Burnout. J Occup Behaviou 1981; 2(2): 99113.

2. Maslach C, Jackson SE, Leiter MP. Maslach Burnout Inventory manual. 3rd ed: Consulting Psychologists Press; 1996.

3. Levert T, Lucas M, Ortlepp K. Burnout in psychiatric nurses: Contributions of the work environment and a Sense of Coherence. S Afr J Psychol 2000; 30(2): 36.

4. Garrosa E, Moreno-Jimenez B, Liang Y, Gonzalez JL. The relationship between socio-demographic variables, job stressors, burnout, and hardy personality in nurses: An exploratory study. Int J Nurs Stud 2008; 45(3): 418-27.

5. Schaufeli WB, Martinez IM, Pinto AM, Salanova M, Bakker AB. Burnout and engagement in university students - A cross-national study. J Cross Cult Psychol 2002; 33(5): 464-81.
6. Batista JBV, Carlotto MS, Coutinho AS, Augusto LGS. Prevalência da síndrome de burnout e fatores sociodemograficos e laborais em professores de escolas municipais da cidade de João Pessoa, PB. Rev Bras Epidemiol 2010; 13(3): 502-12.

7. Salanova MY, Llorens S. Estado actual y retos futuros en el estúdio del Burnout. Papeles del Psicólogo 2008; 5(8): 121-38.

8. McManus IC, Keeling A, Paice E. Stress, burnout and doctors' attitudes to work are determined by personality and learning style: A twelve year longitudinal study of UK medical graduates. BMC Medicine 2004; 2: 29-12.

9. Willcock SM, Daly MG, Tennant CC, Allard BJ. Burnout and psychiatric morbidity in new medical graduates. The Med J Aust 2004; 181(7): 357-60. 
10. Carlotto MS, Nakamura AP, Camara SG. Síndrome de Burnout em estudantes universitários da área da saúde. Psico-USF. 2006;37(1):6.

11. Ried LD, Motycka C, Mobley C, Meldrum M. Comparing Self-reported Burnout of Pharmacy Students on the Founding Campus With Those at Distance Campuses. Am J Pharm Educ 2006; 70(5): 1-12.

12. Barboza JIRA, Beresin R. A síndrome de burnout em graduandos de enfermagem. Einstein 2007; 5(3): 225-30.

13. Maroco J, Tecedeiro M, Martins P, Meireles A. Estrutura fatorial de segunda ordem da Escala de Burnout de Malasch para estudantes numa amostra portuguesa. Análise Psicológica 2008; 4(XXVI): 639-49.

14. Dyrbye LN, Thomas MR, Power DV, Durning S, Moutier C, Massie FS, Jr. et al. Burnout and serious thoughts of dropping out of medical school: a multi-institutional study. Acad Med 2010 Jan; 85(1): 94-102.

15. Zhang Y, Gan Y, Cham H. Perfectionism, academic burnout and engagement among Chinese college students: A structural equation modeling analysis. Personality \& Individual Differences 2007; 43(6): 1529-40.

16. Pöhlmann K, Jonas I, Ruf S, Harzer W. Stress, burnout and health in the clinical period of dental education. Eur J Dental Educ 2005; 9(2): 78-84.

17. Humphris G, Blinkhorn A, Freeman R, Gorter R, HoadReddick G, Murtomaa H, et al. Psychological stress in undergraduate dental students: baseline results from seven European dental schools. Eur J Dental Educ 2002; 6(1): 22-9.

18. Sofola OO, Jeboda SO. Perceived sources of stress in Nigerian dental students. Eur J Dental Educ 2006; 10(1): 20-3.
19. Campos JADB, Zucoloto ML, Bonafé FSS, Jordani PC, Maroco J. Reliability and Validity of self reported burnout in college students: A cross randomized comparison of pencil-and-paper vs. online administration. Comput Hum Behav 2011; 27(5): 1875-83.

20. Carlotto MS, Câmara SG. Características psicométricas do Malasch Burnout Inventory - Student Survey (MBISS) em estudantes universitários brasileiros. Psico-USF 2006; 11(2): 167-73.

21. Maroco J. Análise de equações estruturais. Lisboa: ReportNumber; 2010.

22. Maslach C, Jackson SE. Maslach Burnout Inventory manual. Palo Alto, University of California: Consulting Psychologist Press; 1986.

23. Brasil. Decreto 3048 de 6 de Maio de 1999. In: Social MdPeA, ed. Brasília, DF: Diário Oficial da União; 1999.

24. Alemany Martínez A, Berini Aytés L, Gay Escoda C. The burnout syndrome and associated personality disturbances. The study in three graduate programs in Dentistry at the University of Barcelona. Medicina Oral Patología Oral y Cirurgía Bucal 2008; 13(7): 444-50.

25. Honaker LM. The Equivalency of Computerized and Conventional Mmpi Administration - a Critical-Review. Clin Psychol Rev 1988; 8(6): 561-77.

26. Suris A, Borman PD, Lind L, Kashner TM. Aggression, impulsivity, and health functioning in a veteran population: equivalency and test-retest reliability of computerized and paper-and-pencil administrations. Comput Hum Behav 2007; 23(1): 97-110.

Recebido em: 03/02/11 Versão final apresentada em: 21/06/11 Aprovado em: 08/09/11 